\title{
Detection of soluble ERBB2 in breast cancer cell lysates using a combined label-free/fluorescence platform based on Bloch surface waves
}

\author{
Alberto Sinibaldi, ${ }^{\mathrm{a}^{*}}$ Camilla Sampaoli, ${ }^{\mathrm{b}}$ Norbert Danz, ${ }^{\mathrm{c}}$, Peter Munzert, ${ }^{\mathrm{c}}$ Leonardo Sibilio, ${ }^{\mathrm{d}}$ Frank \\ Sonntag, ${ }^{\mathrm{e}}$ Agostino Occhicone, ${ }^{\mathrm{a}}$ Elisabetta Falvo ${ }^{\mathrm{f}}$, Elisa Tremante, ${ }^{\mathrm{b}}$ Patrizio Giacomini, ${ }^{\mathrm{b}}$ and \\ Francesco Michelotti, ${ }^{\mathrm{a}}$
}

${ }^{a}$ Department of Basic and Applied Science for Engineering, Sapienza University of Rome, Via A. Scarpa 16, 00161 Rome, Italy; ${ }^{b}$ Regina Elena National Cancer Institute, Via Elio Chianesi 53, 00144 Rome, Italy; ${ }^{c}$ Fraunhofer Institute for Applied Optics and Precision Engineering IOF, AlbertEinstein-Str. 7, 07745 Jena, Germany; ${ }^{d}$ IBI- Istituto Biochimico Italiano Giovanni Lorenzini Spa, Via Fossignano 2, 04011 Aprilia, Italy; ${ }^{e}$ Fraunhofer Institute for Material and Beam Technology

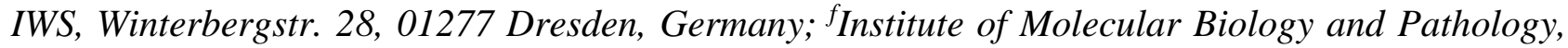
National Research Council, P.le Aldo Moro 5, 00185 Rome, Italy

*corresponding author: alberto.sinibaldi@uniroma1.it

Keywords: Optical biosensors, Bloch surface waves, 1D photonic crystals, ERBB2, SK-BR 3, breast cancer

\begin{abstract}
We report on the use of one-dimensional photonic crystals to detect clinically relevant concentrations of ERBB2/neu/Her2 in cell lysates. ERBB2 is a pivotal breast cancer biomarker and targetable oncogenic driver associated with aggressive breast cancer subtypes. To quantitate soluble ERBB2, we developed an optical platform that combines label-free and fluorescence detection modes. Such platform makes use of a sandwich assay in which the one-dimensional photonic crystals sustaining Bloch surface waves are tailored with a monoclonal antibody for highly specific biological recognition (BSW biochip). In a second step, a second antibody to ERBB2 quantitatively detects the bound analyte. The strategy of the present approach takes advantage of the combination of label-free and fluorescence techniques, making bio-recognition more robust and sensitive. In the fluorescence operation mode, the platform can attain the limit of detection $0.3 \mathrm{ng} / \mathrm{mL}(1.5 \mathrm{pM})$ for ERBB2 in cell lysates. Such resolution meets the international guidelines and recommendations $(15 \mathrm{ng} / \mathrm{mL})$ for diagnostic ERBB2 assays that in the future may help to more precisely assign therapies counteracting cancer cell proliferation and metastatic spread.
\end{abstract}

\section{Introduction}

ERBB2 (also known as Neu, or HER2) is a tyrosine kinase receptor that acts as the master integrator of epidermal growth factor receptor signaling, and regulates a variety of cell proliferation, growth and differentiation pathways. ERBB2 gene amplification/overexpression occurs in approximately $20 \%$ to $30 \%$ of breast cancers and unless treated with ERBB2-targeted therapies this breast cancer subtype is associated with a dismal prognosis (Mahfoud et al., 2014; Negro et al. 2006; Dawood et al. 2010). ERBB2 subtyping, crucial to therapeutic assignment, is routinely carried out by immunohistochemistry and fluorescence in situ histochemistry.

Enzyme-linked immunosorbert assays (ELISA) have been used by several groups to detect soluble ERBB2 since they easily detect baseline levels in the serum of normal subjects and patients carrying non-ERBB2-amplified/overexpressed tumours. This baseline was set at $15 \mathrm{ng} / \mathrm{mL}$ by the US Food and Drug Administration (FDA) in year 2000 (Carney, et al., 2013). Yet, due to the intrinsic 
complexity of cell lysates and body fluids, developing clinical grade biosensors with a sensitivity comparable to ELISA, so as to meet FDA recommendations, remains a challenging biotechnological task. Recently, electrochemical techniques using nanobodies (Patris et al., 2014), nanoelectrode arrays (Mucelli et al., 2008) and amperometric magneto-immunosensor brought new diagnostic insight, leading to limits of detection comparable with ELISA ERBB2 kits (Eletxigerra et al., 2015). In this work, we report on the development of a real-time biosensing platform that takes advantage of both label-free and fluorescence detection and on its use, for the first time, for the assessment of cancer biomarkers in a complex biological matrix, in particular soluble ERBB2 (185kDa and 110kDa) in a cell lysate. This work extends our previous studies, in which we demonstrated the combined label-free and fluorescence operation with preliminary tests based on physical adsorption of fluorescent dyes (Sinibaldi et al., 2014) and label-free detection of a different cancer biomarker (Angiopoietin 2) spiked in a buffer at large concentration (Sinibaldi et al., 2015a, 2015b).

In our setting, the optical transducer is based on a one-dimensional photonic crystal (1DPC) that consists in a dielectric multilayer with suitable refractive index contrast and transparency, supporting Bloch surface waves (BSW) (Shinn et al., 2005; Konopsky et al., 2007). Analogous to surface plasmon polariton biosensing schemes (Wang et al., 2009), the excitation of a Bloch Surface Wave (BSW) can be obtained by a prism coupler leading to a dip in the angular reflectance spectrum. The angular position of such a dip is very sensitive to perturbations and is exploited for bio-sensing applications. Moreover, in presence of fluorescent molecules at the 1DPC surface, the platform can interrogate the BSW biochip also in the enhanced fluorescence mode (Ballarini et al., 2011; Toma et al. 2013), leading to an increase in resolution. Despite the fact that BSW have been claimed to provide better resolution, their validation in a complex biological medium has never been reported until now.

\section{Materials and Methods}

\subsection{Cell biology and biochemistry}

We used two different cell lines: SK-BR-3 and Colo 38. SK-BR-3 breast cancer cells carry an amplified and overexpressed ERBB2 gene, and were used as a convenient source of ERBB2 molecules, since their absolute number per cell $\left(5 \div 6 \times 10^{5}\right.$ molecules per cell) have been concordantly estimated by at least two groups (Shi et al., 2009; Zhang et al., 2011). Colo 38 melanoma cells were selected as a negative control since they do not express detectable amounts of ERBB2 (Galeffi et al., 2005). Details on the cell lines growth, cell characterization and estimated ERBB2 concentration are given in the Supplementary Material (Section S1).

mAbs W6/300G9 (capture Anti-ERBB2) and W6/800E6 (detection Anti-ERBB2) to distinct epitopes of the ERBB2 ectodomain (Digiesi et al. 1992) were used on the biosensing platform for ERBB2 capture and detection, respectively. For fluorescence detection, mAb W6/800E6 was conjugated to the NHS ester of Alexa Fluor 647, at an approximate molar ratio of 10:1 (Anti-ERBB2 AF647).

For internal background referencing mAbs of irrelevant specificity were selected. mAb L 31 binds human Major Histocompatibility Complex class I (MHC I) molecules (Giacomini et al. 1997), and $\mathrm{mAb}$ 34.4.1S binds a highly restricted, polymorphic murine E $\beta$ epitope on MHC II molecules not expressed on any known human protein (Ozato et al., 1982). All mAbs were dissolved in Dulbecco's Phosphate Buffered Saline 1X (D-PBS 1X).

Sulfuric acid (95-98\%), hydrogen peroxide (30\% in $\mathrm{H}_{2} \mathrm{O}$ ), (3-Aminopropyl)triethoxysilane (APTES, $99 \%$ ), ethanol (99.8\%), glutaraldehyde solution (grade I, 50\% in $\mathrm{H}_{2} \mathrm{O}$ ), sodium bicarbonate (99.7\%), sodium cyanoborohydride (95\%), hydrogen chloride (2M), glycine (99\%), bovine serum albumin (BSA, 98\%), and D-PBS 10X (100mM) were purchased from Sigma-Aldrich and used as received. Alexa Fluor 647 NHS Ester $(1 \mathrm{mg} / \mathrm{mL})$ was purchased from Thermo Fisher Scientific. 


\subsection{Bloch surface wave biochip}

The basic element of the Bloch surface wave (BSW) biochip is a one dimensional photonic crystal (1DPC) that is designed to sustain electromagnetic surface waves confined at its surface. The 1DPC is deposited on a glass substrate and is constituted by an optical multilayer with a combination of low and high refractive index dielectric materials. The localization of BSW at the interface between a finite 1DPC and an external dielectric medium, generally an aqueous solution, is guaranteed by Bragg reflection and total internal reflection on the two sides of the interface, respectively (Yeh et al. 1977; Shinn et al. 2005).

The 1DPC were deposited on glass microscope slides by plasma ion assisted deposition (PIAD) under high vacuum conditions (Leybold Optics, APS904) (Sinibaldi et al., 2015a). The dielectric materials were silica $\left(\mathrm{SiO}_{2}\right)$ for the low index layers, and tantala $\left(\mathrm{Ta}_{2} \mathrm{O}_{5}\right)$ and titania $\left(\mathrm{TiO}_{2}\right)$ for the high index layers. The refractive indices at $\lambda_{\mathrm{LF}}$ were determined either by reflection /transmission spectroscopy on single layers or by ellipsometry: $\mathrm{n}\left(\mathrm{SiO}_{2}\right)=1.474+\mathrm{j} 5 \times 10^{-6}$ and $\mathrm{n}\left(\mathrm{Ta}_{2} \mathrm{O}_{5}\right)=2.160+\mathrm{j} 5 \times 10^{-5}$, $\mathrm{n}\left(\mathrm{TiO}_{2}\right)=2.28+\mathrm{j} 1.8 \times 10^{-3}$. Starting from the substrate, the 1DPC consists of a stack with a first silica layer, a periodic part with two tantala/silica bilayers and a topping thin titania/silica bilayer. The nominal thicknesses are $\mathrm{d}\left(\mathrm{SiO}_{2}\right)=275 \mathrm{~nm}, \mathrm{~d}\left(\mathrm{Ta}_{2} \mathrm{O}_{5}\right)=120 \mathrm{~nm}$ for the periodic part and $\mathrm{d}\left(\mathrm{TiO}_{2}\right)=20$ $\mathrm{nm}, \mathrm{d}\left(\mathrm{SiO}_{2}\right)=20 \mathrm{~nm}$ for the topping layers.

On the sensitive surface of the BSW biochip one can define several spots along the $\mathrm{x}$ direction (indicated with S, R1 and R2 in Figure 1a), where different capture mAbs (either specific or nonspecific) are immobilized. The sensing spots are probed simultaneously by a parallel read-out system based on strip-shaped illumination beams for both label-free and fluorescence operation modes (Figures 1a and 1d), which are obtained by using cylindrical optics. Detection is carried out by means of a cylindrical optical system and a CCD array detector that provides along one direction (columns) the angular dependence of the intensity for each position $\mathrm{x}$ (rows direction).

a)

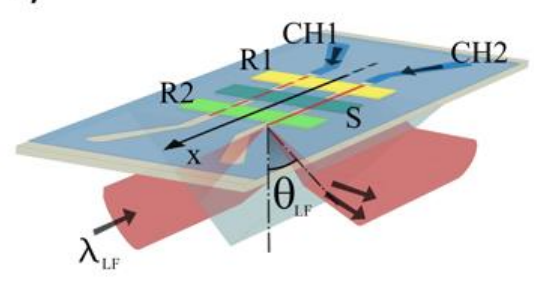

d)

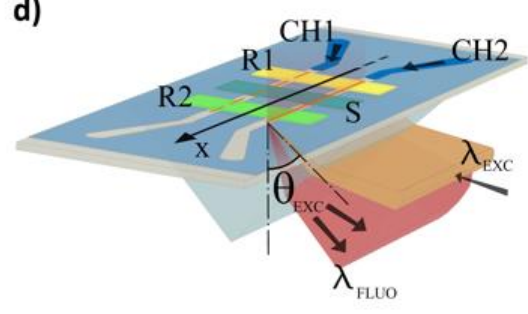

b)
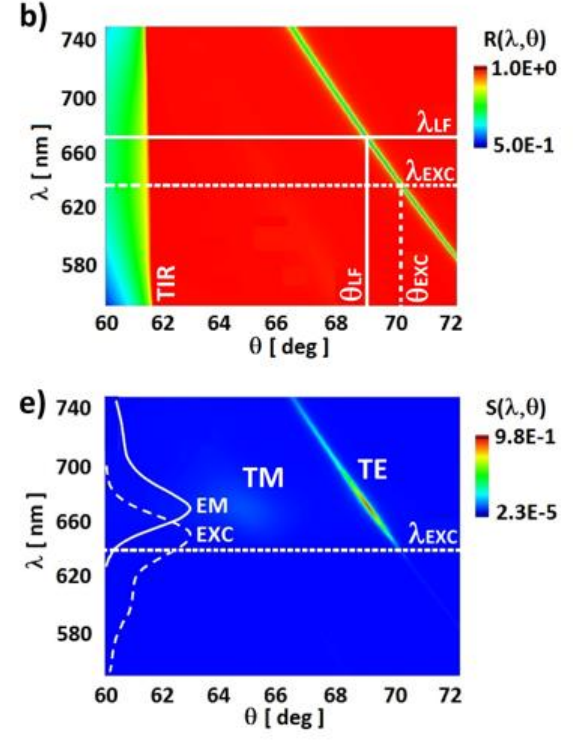

c)
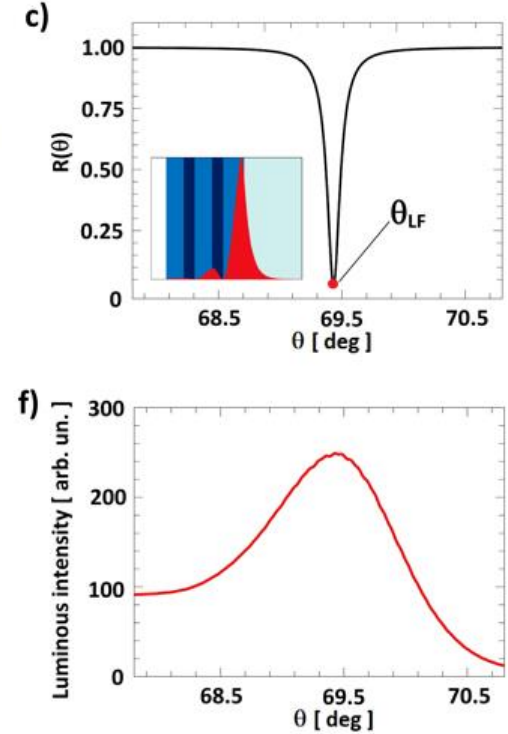

Figure 1: (a) Optical layout for the LF mode. $\mathrm{CH} 1$ and $\mathrm{CH} 2$ are the two-channels of the fluidic cell; $\mathrm{S}$ and $R 1, R 2$ are one signal and two reference spots, respectively (b) Reflectance map $R(\lambda, \theta)$ calculated for the design 1DPC and TE polarization. $\lambda_{\mathrm{LF}}$ and $\lambda_{\mathrm{EXC}}$ are the lasers' wavelengths; $\theta_{\mathrm{LF}}$ and $\theta_{\mathrm{EXC}}$ are the corresponding resonance angles,. (c) $\mathrm{R}(\theta)$ at $\lambda_{\mathrm{LF}}$ for the TE polarization. Inset: geometry of the 1DPC and BSW intensity distribution at resonance. (d) Optical layout for the FLUO mode. Excitation at $\lambda_{\text {EXC }}$ and fluorescence collection at $\lambda_{\text {FLUO }}$ take place from the same side. (e) Emitted intensity map $S(\lambda, \theta)$ calculated for isotropically oriented Alexa Fluor 647 dye molecules at the surface of the 1DPC, for both TE and TM (weak) polarizations. The dye absorption and emission spectra are shown on the left axis. (f) Radiant intensity $\mathrm{I}(\theta)$ calculated for isotropically oriented dye molecules at the surface of the 1DPC. 
We designed and fabricated a microfluidic cell with two channels (indicated with $\mathrm{CH} 1$ and $\mathrm{CH} 2$ in Figure 1a) that are aligned to the strip-shaped focused beam (red line parallel to $\mathrm{CH} 2$ in Figure 1a) and permit to perform two assays on the same chip in a sequence.

For label-free operation (LF mode), a cylindrically focused ( $\Delta \theta \sim 5 \mathrm{deg}$ ) laser beam at $\lambda_{\mathrm{LF}}=670 \mathrm{~nm}$ is coupled to the biochip by means a glass prism (Figure 1a). Owing to the illumination configuration (Danz et al. 2011), the angular reflectance $R\left(\lambda_{L F}, \theta\right)$ is probed simultaneously in every spot along $x$ (Sinibaldi et al., 2015a). In Figure $1 \mathrm{~b}$ we show the calculated $\mathrm{R}(\lambda, \theta)$ map for the TE polarization case, where the BSW dispersion $\lambda(\theta)$ appears as a dark line beyond the total internal reflection (TIR) edge. When operating at fixed $\lambda_{\mathrm{LF}}$ a dip at $\theta_{\mathrm{LF}}$ is observed in the $\mathrm{R}(\theta)$ profile (Figure $1 \mathrm{c}$ ). By tracking $\theta_{\mathrm{LF}}$ on the CCD images one can therefore probe, spot by spot, the refractive index changes and the amount of analytes captured at the biochip surface. The accuracy of the determination of $\theta_{\mathrm{LF}}$ depends both on the 1DPC design and on the optical detection system, and was deeply investigated in our previous work (Rizzo et al., 2014).

For fluorescence operation (FLUO mode), a laser beam at $\lambda_{\mathrm{EXC}}=635 \mathrm{~nm}$ is cylindrically and weakly focused $(\Delta \theta \sim 1 \mathrm{deg})$ through the prism at $\theta_{\mathrm{EXC}}$ in order to couple to the BSW at $\lambda_{\mathrm{EXC}}$ and to resonantly excite dye molecules at the surface (Figure 1d). $\lambda_{\text {EXC }}$ matches the maximum of the dye absorption spectrum (Figure 1e). The angular emission of the dye molecules in the presence of the 1DPC is modified and re-directed in the collecting system (Ballarini et al., 2011). In Figure 1e, we show the intensity $S(\lambda, \theta)$ emitted in the prism by randomly oriented dye labels at the 1DPC surface. The map was calculated for the Alexa Fluor 647 dye by means of a rigorous electrodynamic approach applying dyadic Green's functions to describe the effects classically (Danz et al., 2002). Each $\lambda$ component of the dye spectrum is emitted at a different angle $\theta$ according to the BSW dispersion $\lambda(\theta)$ for both the TE and TM polarizations. A wavelength insensitive sensor will measure the integral of $\mathrm{S}(\lambda, \theta)$ over $\lambda$, i.e. the radiant intensity $\mathrm{I}(\theta)$, which is shown in Figure $1 \mathrm{f}$.

\subsection{Bio-conjugation strategy}

The bare BSW biochips were first cleaned in a piranha solution (3:1 mixture of sulfuric acid and 30 $\%$ hydrogen peroxide) for 10 minutes. The biochips were then rinsed thoroughly with de-ionized water and dried under a stream of nitrogen gas, to remove of all organic contaminants and expose hydroxyl groups for the following functionalization step. The clean biochips were immersed into a 2 $\%$ solution of APTES in ethanol/water $(95: 5 \mathrm{v} / \mathrm{v})$ mixture at room temperature $(\mathrm{RT})$ for $1 \mathrm{~h}$. The chips were then removed from the APTES solution, sonicated, rinsed with ethanol and baked on a hot plate at $110{ }^{\circ} \mathrm{C}$ for $1 \mathrm{~h}$. The APTES-modified chips were allowed to react with $1 \%(\mathrm{v} / \mathrm{v})$ glutaraldehyde in $100 \mathrm{mM}$ sodium bicarbonate buffer ( $\mathrm{pH} \mathrm{8.5)}$ in the presence of $0.1 \mathrm{mM}$ sodium cyanoborohydride for $1 \mathrm{~h}$ at RT. A second sonication and rinsing in de-ionized water were performed.

The glutaraldehyde-activated surface of the biochip was then divided, by means of a hydrophobic marker, into signal and reference spots where either ERBB2 specific or non-specific mAbs were incubated. In all cases we dissolved the mAbs in D-PBS $1 \mathrm{X}$ at $0.5 \mathrm{mg} / \mathrm{ml}$ and incubated for 1 hour at RT. After a further washing step, the biochips were immersed in a solution of BSA $(10 \mathrm{mg} / \mathrm{ml})$ in DPBS $1 \mathrm{X}$ to block the remaining reactive sites (overnight at $4{ }^{\circ} \mathrm{C}$ ). At the end the biochip was washed in D-PBS $1 X$.

\subsection{Optical read out system}

Figure 2 shows the scheme of the optical read out system. Before its use, the bio-conjugated BSW biochips' surface is topped with the two-channels fluidic cell and the back face is coupled to a BK7 glass prism by means of a refractive index matching oil. Then the biochip is mounted in the optical platform and is topped by an aluminium back plate with a PDMS contact layer that provides the fluidic connections. Translating the coupling prism one can use either $\mathrm{CH} 1$ or $\mathrm{CH} 2$. Details on the 
fluidic cell fabrication and fluid handling system are given in the Supplementary material (Section S2).

For the LF mode, a temperature stabilized $\left( \pm 0.01^{\circ} \mathrm{C}\right)$ laser diode $(\mathrm{LD} 1)$ at $\lambda_{\mathrm{LF}}$ (Thorlabs LPS-675FC) is collimated and TE polarized by means a polarizer (POL). The beam is expanded by means of a telescope and the central portion is selected by a circular diaphragm (D). The parallel beam is focused by a cylindrical lens $\left(f_{1}=100 \mathrm{~mm}\right.$ ) onto the coupling prism. Rotation stages are used to set the average incidence angle at $\theta_{\mathrm{LF}}$ and the angle of the detection arm at $2 \theta_{\text {LF. }}$ The reflected beam is imaged by a cylindrical Fourier lens $\left(\mathrm{f}_{2}=75 \mathrm{~mm}\right.$ ) onto a monochrome CCD camera (Apogee Ascent, Sony ICX814 chip, $\mathrm{W}=3388$ pixel, $\mathrm{H}=2712$ pixel, corresponding to $12.50 \mathrm{~mm}$ and $10.00 \mathrm{~mm}$, respectively). The long dimension $\mathrm{W}$ of the $\mathrm{CCD}$ is used to image the angular reflectance and achieve best sampling of the BSW resonance, with a 2.7 deg field of view, which is determined by W and $\mathrm{f}_{2}$. The conversion factor between pixels and angle is 7.9E-4 deg / pix. The spots along the $\mathrm{x}$ direction are imaged along the short dimension $\mathrm{H}$ of the CCD by means of a properly positioned cylindrical lens $\left(f_{3}=150 \mathrm{~mm}\right)$. A rotating scatterer $(\mathrm{RS})$, placed inside the telescope, destroys the spatial coherence of the illumination beam and the CCD integration time is set to integrate the scattered light, thus ruling out speckles' effect.

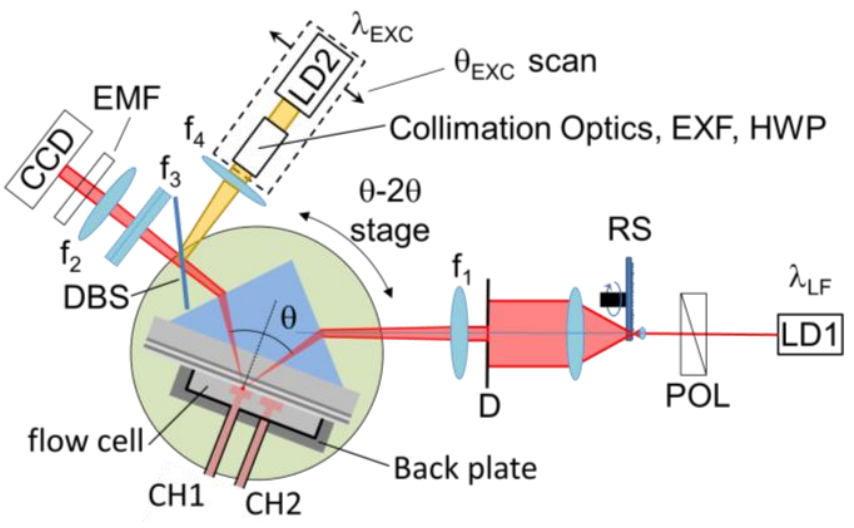

Figure 2: Simplified layout of the optical setup used to interrogate the BSW biochips.

The FLUO mode is implemented by making use of the same collection optics and CCD sensor used for the LF mode (different integration time and binning) (Sinibaldi et al., 2014) and introducing a second laser for the labels' excitation. A polarized laser diode emitting at $\lambda_{\text {EXC }}$ (LD2) is collimated and focused by a cylindrical lens $\left(\mathrm{f}_{4}=130 \mathrm{~mm}\right)$ to a strip on the chip surface. A dichroic beam splitter (DBS, Chroma ZT 640 RDC) is used to reflect the excitation beam and transmit fluorescence emission. The collimation optics, the excitation filter (EXF, Chroma ZET 635/20) and the half wave plate (HWP) used to set the polarization to TE are arranged inside the excitation arm. Great care was taken to align the fluorescence excitation and the label-free laser strips. The average incidence angle $\theta_{\text {EXC }}$ can be tuned by translating the whole fluorescence excitation LD2 module (dashed box in Figure 2). An emission filter (EMF, Chroma 655 LP ET Longpass Filter) in front of the CCD cuts stray light from the excitation beam; such filter transmits at $\lambda_{\mathrm{LF}}$, therefore preserving the label-free operation. The design of the optical detection system ensures that the same angular range is observed for all spots along the illuminated region on the sensor surface for both LF and FLUO modes.

\section{Results}

The procedures used in the cancer biomarkers detection assays were developed in preliminary experiments carried out with recombinant ERBB2-Fc fusion chimera (R\&D Systems) spiked in DPBS 1X. Details on the optimized protocols developed and on the results obtained with ERBB2-Fc 
are given in the Supplementary material (Section S3). Before starting an assay the biochip's surface is regenerated and the sensitivity in each spot is measured according to the procedures described in the Supplementary material (Section S4).

For the ERBB2 detection assays in cell lysates we made use of the samples listed in Table I. Dilutions of SK-BR-3 and Colo 38 lysates were prepared in lysis buffer at 1:16, 1:40, 1:160 and 1:800, giving rise to four ERBB2 positive (from P1 to P4) and four ERBB2 negative samples (from N1 to N4). In Table I, we list the total protein concentration $\mathrm{C}_{\mathrm{WH}}$, the ERBB2 concentration $\mathrm{C}_{\mathrm{ERBB} 2}$ and the mass of the raw lysates $\mathrm{m}_{\mathrm{LYS}}$ for all dilutions.

\begin{tabular}{|c|c|c|c|c|c|}
\hline SAMPLE & Cell line & Dilution & $\begin{array}{c}\mathrm{CWH}_{\mathrm{WH}} \\
{[\mu \mathrm{g} / \mathrm{mL}]}\end{array}$ & $\begin{array}{c}\text { CERBB2 } \\
{[\mathrm{ng} / \mathrm{mL}]}\end{array}$ & $\begin{array}{r}\mathbf{m}_{\mathrm{LYS}} \\
{[\mu \mathrm{g}]}\end{array}$ \\
\hline P1 & \multirow{4}{*}{ SK-BR-3 } & $1: 16$ & 500 & 175 & 60 \\
\hline $\mathrm{P} 2$ & & $1: 40$ & 200 & 70 & 24 \\
\hline P3 & & $1: 160$ & 50 & 17.5 & 6 \\
\hline $\mathrm{P} 4$ & & $1: 800$ & 10 & 3.5 & 1.2 \\
\hline N1 & \multirow{4}{*}{ Colo 38} & $1: 16$ & 500 & - & 60 \\
\hline N2 & & $1: 40$ & 200 & - & 24 \\
\hline N3 & & $1: 160$ & 50 & - & 6 \\
\hline N4 & & $1: 800$ & 10 & - & 1.2 \\
\hline
\end{tabular}

Table I - Lysate samples used in the assays carried out with the optical biosensing platform.

We defined on the biochip three spots (S, R1, and R2 in Figure 1a), where either capture Anti-ERBB2 (signal, S), or reference Anti-MHC II (R1) and Anti-MHC I (R2) were bio-conjugated. Each biochip were used for two assays in a sequence; the first with a SK-BR-3 sample in CH1 and the second with a Colo 38 sample in $\mathrm{CH} 2$ at identical dilutions (same $\mathrm{C}_{\mathrm{wH}}$ ). The two assays were performed with a temporal delay of 1 hour and no degradation of the performances was observed within such a timeframe. Such a strategy guarantees that the functionalization chemistry and immobilization steps were the same for the two channels and lysate solutions. In Figure 3a, we show the results of the LF assay carried out with one BSW biochip for the P1 sample ( $\mathrm{CH} 1)$. The curves correspond to the sensograms registered in the S (black), R1 (red) and R2 (blue) spots. In the inset we show the last part of the sensogram (detection mAb) registered for the N1 control solution $(\mathrm{CH} 2)$.

For all BSW biochips the assay was carried out as follows. At the beginning the fluorescence background was measured in FLUO mode in D-PBS 1X, then the platform was switched to the LF mode and started to record the sensograms. The lysate solution $(120 \mu \mathrm{L})$ was injected and recirculated during incubation (12 min). The biochip was then washed with D-PBS 1X. The labelled detection antibody Anti-ERBB2 AF647 $(120 \mu \mathrm{L})$ was injected and recirculated during incubation (15 min). Finally the biochip is washed with D-PBS $1 \mathrm{X}$ and the fluorescence is measured in FLUO mode. Starting from the lysate injection the duration of the assay is $30 \mathrm{~min}$. 

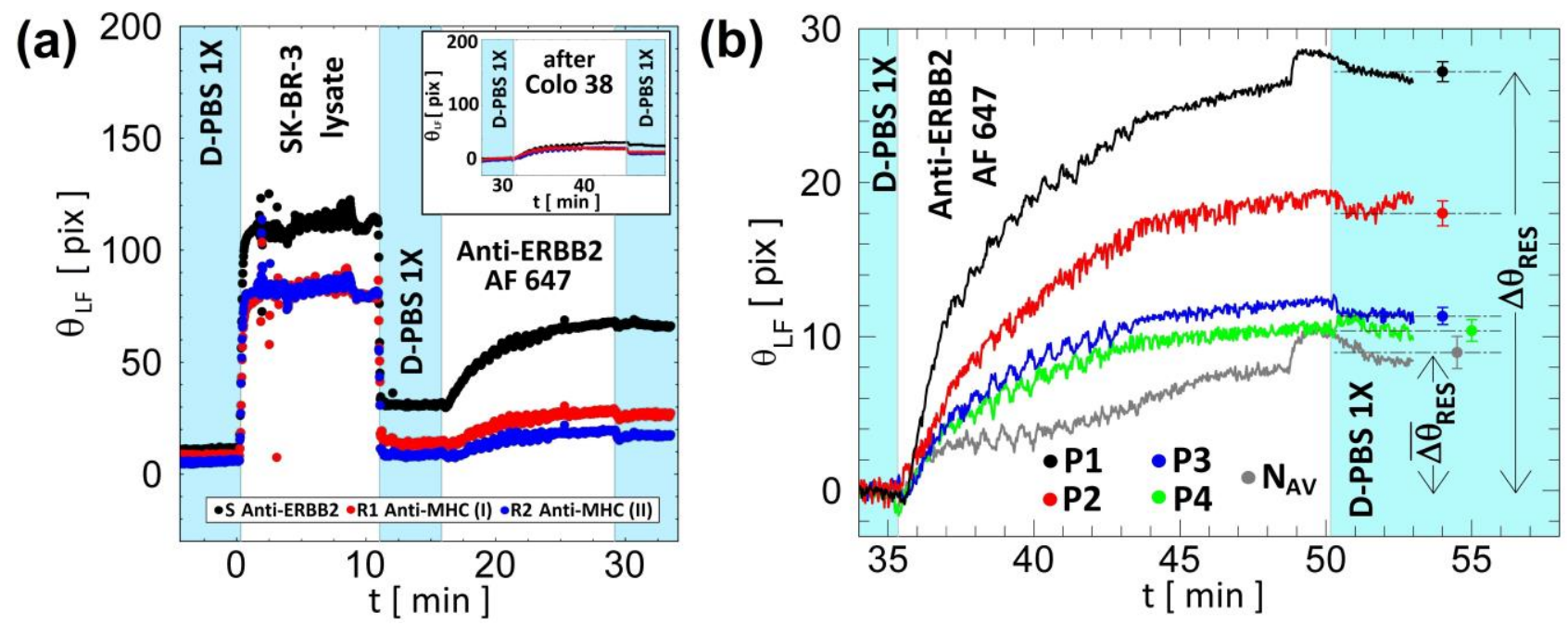

Figure 3: (a) LF signal recorded during a complete ERBB2 bio-recognition assay in a SK-BR-3 cell lysate (sample P1). The three sensograms correspond to the specific (black) and non-specific (red and blue) spots where the capture mAbs were incubated. (Inset) LF signal recorded during incubation with the detection antibody for the same biochip in the $\mathrm{CH} 2$ and after exposing the biochip to the Colo 38 cell lysate (sample N1). The colour codes of the curves are the same as in the main figure. (b) (Curve P1 to P4) Anti-ERBB2 AF647 binding kinetics for different $\mathrm{C}_{\mathrm{ERBB} 2}$. $\left(\mathrm{N}_{\mathrm{AV}}\right)$ Colo 38 average signal for three $\mathrm{BSW}$ biochips (grey). The error bars $(3 \sigma)$ refer to the average residual shifts calculated in D-PBS $1 \mathrm{X}$ at the end of the assay. $\Delta \theta_{R E S}$ is the residual shift for the $\mathrm{P}$ samples and $\overline{\Delta \theta}_{R E S}$ is the average residual shift for the $\mathrm{N}$ samples.

Figure 3a shows that specific binding of ERBB2 to the $\mathrm{S}$ spot takes place, whereas a reduced response due to non-specific binding is observed in the R1 and R2 spots. A residual shift is observed after lysate incubation and wash with D-PBS $1 \mathrm{X}$, which is amplified after the detection mAb incubation step. The signals recorded during the detection $\mathrm{mAb}$ incubation are much cleaner, due to the decreased complexity of the matrix, and it is possible to track the differential signal and the binding kinetics. Such result suggests that tracking the LF signals during the incubation with the detection antibody can give information that is much more reliable and can extend the standard label-free direct binding assays (Eletxigerra et al., 2016). In the inset of Figure 3a we show, for the N1 solution injected in $\mathrm{CH} 2$, the sensograms recorded during the incubation with the detection $\mathrm{mAb}$. The response of all spots is low when ERBB2-negative Colo 38 lysates are added, demonstrating the ERBB2 specificity of the assay.

In Figure $3 b$ we report the differential LF sensograms, during the Anti-ERBB2 AF647 injection $(120 \mu \mathrm{L})$ and incubation/recirculation, for four identical BSW biochips that were previously exposed to dilutions (P1 to P4) of the SK-BR-3 lysate. The differential curves are normalized by subtracting the signals recorded in the spots $\mathrm{S}$ and $\mathrm{R} 1$, respectively. All curves were obtained under the same experimental conditions. The binding kinetics and residual shifts $\Delta \theta_{\text {RES }}$ after washing with D-PBS $1 \mathrm{X}$ can be clearly observed. The assays carried out with the Colo 38 lysate solutions (N1 to N4), show binding kinetics and residual signals (Figure S6) that are always below the P4 dilution (smallest ERBB2 content). In Figure $3 \mathrm{~b}$ we plot the curve obtained by averaging the differential sensograms for three Colo 38 lysate solutions, which shows a residual average shift $\overline{\Delta \theta}_{R E S}$.

In Figure 4, we show the results of the measurements carried out in FLUO mode for the same assays that provided the LF results shown in Figure $3 b$ and Figure S6. The background subtracted radiant intensity $\mathrm{I}(\theta)$ recorded by the CCD camera for each spot and for each lysate solution (see for example Figure $2 \mathrm{f}$ and Figure S3 in the Supplementary material) was integrated over the angular detection window, providing a the total emitted power $\mathrm{W}$. The histogram summarizes the $\mathrm{W}$ values obtained 
for all cases. We observe a marked difference between the $\mathrm{W}$ values measured for the ERBB2 positive and negative cell lines and between the different sensing spots. In the inset of Figure 4, we show, for the greatest dilution and for the three sensing spots, the difference of fluorescence powers $\mathrm{W}(\mathrm{P} 4)-$ W(N4) recorded for the SK-BR-3 and Colo 38 samples, respectively. This shows that, even at the lowest ERBB2 content the assay is able to distinguish between a positive and a negative control.

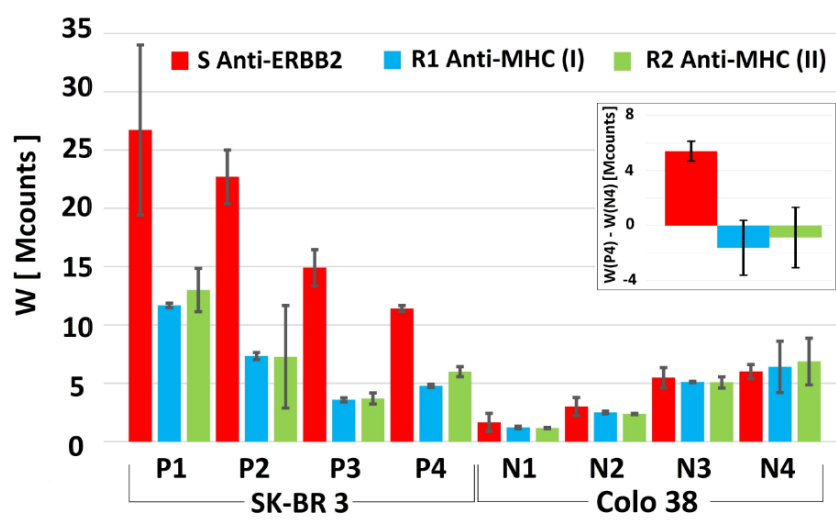

Figure 4: Background subtracted power $\mathrm{W}$ after exposition to Anti-ERBB2 AF647 and for different SK-BR3 and Colo 38 samples. The different colours denote fluorescence values obtained for the three sensitive spots on the BSW biochips. (Inset) W(P4)-W(N4) for the three spots.

\section{Analysis and Discussion}

The results show that the biosensing platform can operate efficiently, integrating both the LF and FLUO modes and making use of the same BSW biochips and optical read out. The platform detected ERBB2 in a complex matrix in the FLUO mode at the clinically relevant concentration of $3.5 \mathrm{ng} / \mathrm{mL}$ (19 pM). In serum, a less complex biological matrix with respect to cell lysates, the well-established clinical usefulness threshold for ERBB2 is placed at $15 \mathrm{ng} / \mathrm{mL}$ (Carney, et al., 2013). In the LF mode, the platform detected ERBB2 at a concentration of $17.5 \mathrm{ng} / \mathrm{m}$ that is in the range of but not below this threshold. Nevertheless, the performance in the LF is improved with respect to results of previous works with LF and FLUO combined platforms, i.e. 60-100 ng/mL in spiked samples (Michelotti et al., 2016; Wang et al., 2009).

Below we analyse data more in detail and evaluate the limit of detection (LoD), i.e. the minimum ERBB2 concentration that can be detected in a lysate solution by the immunoassay implemented on the platform, either in the LF or FLUO modes.

In Figure 5a, we report the result of the analysis of the LF data shown in Figure 3b. For each SK-BR3 lysate dilution, we calculated the difference $\Delta \theta_{N}=\Delta \theta_{R E S}-\overline{\Delta \theta}_{R E S}$ and plot it as a function of $\mathrm{C}_{\text {ERBB2. }}$ We found that the Langmuir isotherm model does not fit to the experimental data. However, we can interpolate by means of the Hill's model (Morales et al., 1999):

$$
\Delta \theta_{N}=\Delta \theta_{0}+\frac{\Delta \theta_{\text {sat }}-\Delta \theta_{0}}{1+\left(K_{D} / c\right)^{\beta}}
$$

where $\Delta \theta_{0}$ is a residual plateau level, $\Delta \theta_{\text {sat }}$ is the saturation level, $K_{D}$ is the equilibrium dissociation constant of ERBB2 to the capture Anti-ERBB2 and $\beta$ is a non-ideality parameter $\left(\Delta \theta_{0}=0.62\right.$ pixel, $\Delta \theta_{\text {sat }}=21.9$ pixel, $\mathrm{K}_{\mathrm{D}}=95 \mathrm{ng} / \mathrm{mL}=0.5 \mathrm{nM}, \beta=1.9$ ). Assuming that a signal can be detected if it differs from $\Delta \theta_{0}$ by more than the standard deviation, we obtain that $\mathrm{LoD}_{\mathrm{LF}}=14.5 \mathrm{ng} / \mathrm{mL}=78 \mathrm{pM}$. 


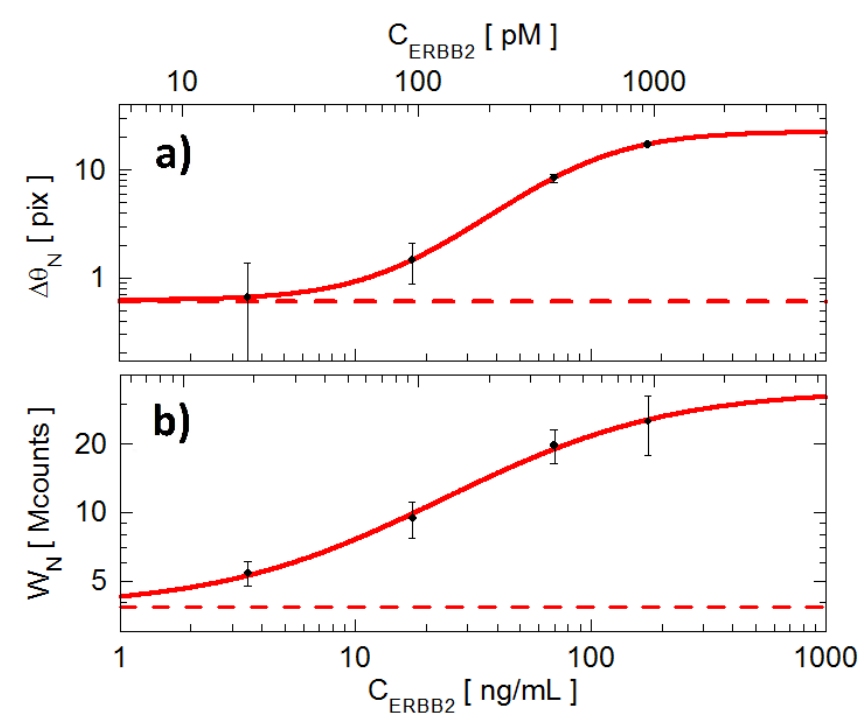

Figure 5: Calibration curves for assays carried out with ERBB2 expressing cell lysates. The error bars show the standard deviation. (a) LF data interpolated with Eq.(1) (b) FLUO data fitted with Eq.(2). The dashed horizontal lines correspond to the $\Delta \theta_{0}$ and $\mathrm{W}_{0}$ values.

In Figure 5b, we report the result of the analysis of the FLUO data shown in Figure 4. We plot the difference $\mathrm{W}_{\mathrm{N}}$ between the $\mathrm{W}$ values found in the signal spot $(\mathrm{S})$, for BSW biochips exposed to either SK-BR-3 or Colo 38 samples, as a function of CERBB2. We fitted data by means of a modified Langmuir isotherm model, taking into account a background signal $\mathrm{W}_{0}$ (O'Shannessy et al., 1996):

$$
W_{N}=W_{0}+\frac{W_{s a t}-W_{0}}{1+K_{D} / c}
$$

where $\mathrm{W}_{\text {sat }}$ is the saturation $\left(\mathrm{W}_{\text {sat }}=30.3\right.$ Mcounts, $\mathrm{W}_{0}=3.8$ Mcounts, $\left.\mathrm{K}_{\mathrm{D}}=(70 \pm 25) \mathrm{ng} / \mathrm{mL}=(0.3 \pm 0.1) \mathrm{nM}\right)$.

$\mathrm{K}_{\mathrm{D}}$, which is in the range the value interpolated from the LF data, is among the smallest found for state-of-the-art high ERBB2 affinity proteins $\left(0.3 \mathrm{nM}<\mathrm{K}_{\mathrm{D}}<5.8 \mathrm{nM}\right)$ developed for cancer therapy (Nilvebrant et al., 2014). The value is also consistent with the estimation (1 nM) obtained using radiobinding assays on cultured cells (Digiesi et al., 1992 and unpublished results). Of course, evaluating $K_{D}$ from Langmuir-like plot may suffer from a great uncertainty. Kinetic measurements of the association $\left(\mathrm{k}_{\mathrm{a}}\right)$ and dissociation $\left(\mathrm{k}_{\mathrm{d}}\right)$ constants and of their ratio $\mathrm{K}_{\mathrm{D}}=\mathrm{k}_{\mathrm{d}} / \mathrm{k}_{\mathrm{a}}$ would be preferable and is one of the objectives of our future work. However, the value found for $\mathrm{K}_{\mathrm{D}}$ already gives a strong indication that the Anti-ERBB2 capture antibody used in this work is extremely avid for ERBB2.

Assuming that a signal can be detected if it differs from $\mathrm{W}_{0}$ by more than the standard deviation, we find that $\mathrm{LoD}_{\mathrm{FLUO}}=(1.7 \pm 0.7) \mathrm{ng} / \mathrm{mL}=(9 \pm 4) \mathrm{pM}$, showing that in the FLUO mode the LoD is about 10 times smaller than the LF case. Such a LoD ${ }_{\text {FLUO }}$ is well below the standard immunohistochemistry limits and in the range of the ELISA commercial kits for ERBB2 $(0.2 \mathrm{ng} / \mathrm{mL}$, www.thermofisher.com).

As far as precision of the assay is concerned, from Figure 5, we can evaluate that around the lowest measured ERBB2 concentration $(3.5 \mathrm{ng} / \mathrm{mL})$ the precision on the determination of the concentration from a single FLUO measurement in one spot is $50 \%$, and decreases to $30 \%$ around the $15 \mathrm{ng} / \mathrm{mL}$ ERBB2 threshold. In the LF mode such precision is $50 \%$ around the LoD $\mathrm{LF}_{\mathrm{LF}}$

The LoD FLUO we found is smaller than all other results reported in literature for ERBB2 with platforms which can be considered for point-of-care applications, say with an assay duration shorter than 2 hours (Eletxigerra et al., 2015 and references therein). In our case the duration of the assay is only $30 \mathrm{~min}$, positioning the BSW biochip as a top-ranking device in terms of assay response time, outperforming standard ELISA kits, whose total assay time is approximately 4 hours. 
We remark that, if the data shown in Figure 5 are analysed according to other procedures that are commonly reported in literature (Wang et al., 2009; Vaisocherová et al., 2006), the LoD is smaller. According to such approaches, we linearly fitted data and evaluated the LoD as the concentration for which the fit reaches 3 times the standard deviation of the error $\sigma$ (Figure S7, Supplementary material). We get $\sigma_{\mathrm{LF}}\left(\Delta \theta_{\mathrm{N}}\right)=0.7 \mathrm{pix}$ and $\operatorname{LoD}_{\mathrm{LF}}=(16 \pm 9) \mathrm{ng} / \mathrm{mL}=(90 \pm 50 \mathrm{pM})$ and $\sigma_{\text {FLUO }}\left(\mathrm{W}_{\mathrm{N}}\right)=0.65$ Mcounts and $\operatorname{LoD}_{\text {FLUO }}=(0.3 \pm 0.2) \mathrm{ng} / \mathrm{mL}=(1.5 \pm 1.0) \mathrm{pM}$. Such values should be used to compare the resolution of our platform to other systems that were calibrated according to such a procedure.

Figure 5 suggests that the LoD could be improved by optimizing the blocking step. An insight on the blocking efficiency can be indirectly found by observing in detail Figure 4. The Colo 38 results show that increasing values of $\mathrm{W}$ (for all spots $\mathrm{S}, \mathrm{R} 1$ and $\mathrm{R} 2$ ) are found when the complexity of the matrix decreased, i.e. from $\mathrm{N}_{1}$ to $\mathrm{N}_{4}$, indicating that increasing lysate concentration improves the passivation. The lysate matrix contributes to blocking, playing a role in the non-specificity of the surface.

We point out that the LoD $D_{L F}$ for ERBB2 spiked in D-PBS $1 \mathrm{X}$ is indeed lower, as expected due to the reduced complexity of the matrix. We find $\mathrm{LoD}_{\mathrm{LF}}=2.3 \mathrm{ng} / \mathrm{mL}=10 \mathrm{pM}$ (Supplementary material, Section III).

\section{Conclusions}

An optical biochip and the read out system for recognition of ERBB2 in cell lysates were developed by using one dimensional photonic crystals sustaining Bloch surface waves as a transducer. A sensitive immunoassay was implemented and optimized. The analytical characteristics, in terms of limit of detection, of the proposed ERBB2 assay brings an improvement with respect to other biosensors (Al-Khafaji et al., 2012; Marques et al., 2014; Mucelli et al., 2008) reaching a LoD of $1.7 \mathrm{ng} / \mathrm{mL}(0.3 \mathrm{ng} / \mathrm{mL}$ if assessed with a linear extrapolation). Furthermore, the presented BSW biochip shows a LoD in the range of an ERBB2 commercial ELISA kit, with a considerable difference in the assay duration, only 30 minutes in this case. The resolution achieved is actually adequate for the analysis of serum sample, since $15 \mathrm{ng} / \mathrm{mL}$ was defined as the appropriate threshold between basal and abnormal ERBB2 protein expression levels.

In addition, the possibility to assay molecules using a platform that ensures two different quantification techniques (LF or FLUO modes), while maintaining the optical system unchanged, is the real added value of the study. Combined detection in real-time introduces a clear advantage in terms of result reliability to clinical diagnosis. Future investigations will be devoted to human plasma samples trying to apply directly the described technique to real world studies.

\section{Acknowledgements}

Thanks are expressed to IBI- Istituto Biochimico Italiano Giovanni Lorenzini for logistic help and especially to Rocco Fraioli from Regina Elena National Cancer Institute for skilful technical assistance. This work was partially funded by the European Commission through the project BILOBA (Grant Agreement 318035), and by AIRC IG grant 14204 to PG.

\section{References}

Al-Khafaji, Q. A. M., Harris, M., Tombelli, S., Laschi, S., Turner A. P. F., Mascini, M., and Marrazza, G. 2012. Electroanal., 24, 4, 735-742.

Ballarini, M., Frascella, F., Michelotti, F., Digregorio, G., Rivolo, P., Paeder, V., Musi, V., Giorgis, F. and Descrovi, E., 2011. Appl. Phys. Lett., 99, 4, 043302. 
Carney, W.P., Bernhardt, D., and Jasani, B., 2013. Biomark Cancer 5, 31-39.

Danz, N., Waldhäusl, R., Bräuer, A., and Kowarschik, R., 2002. J. Opt. Soc. Am. B, 19, 3, 412-419.

Danz, N., Kick, A., Sonntag, F., Schmieder, S., Höfer, B., Klotzbach, U., Mertig, M., 2011. Eng. Life Sci., 11, 566-572.

Dawood, S., Broglio, K., Buzdar, A.U., Hortobagyi, G.N., Giordano, S.H.., 2010. J. Clin. Oncol.. 28(1):92-98.

Digiesi, G., Giacomini, P., Fraioli, R., Mariani, M., Nicotra, M.R., Segatto, O., et al., 1992. Hybridoma; 11, 519-527.

Eletxigerra, U., Martinez-Perdiguero, J., Merino, S., Barderas, R., Torrente-Rodríguez, R.M., Villalonga, R., Pingarrón, J.M., Campuzano, S., 2015. Biosens Bioelectron. 15;70:34-41.

Eletxigerra, U., Martinez-Perdiguero, J., Barderas, R., Pingarrón, J.M., Campuzano, S., Merino, S., 2016. Anal. Chim. Acta, 905, 156-162.

Galeffi, P., Lombardi, A., Donato, M.D., Latini, A, Sperandei, M., Cantale, C., Giacomini, P., 2005. Vaccine, 23:1823-1827.

Giacomini, P., Ng, A.K., Kantor, R.R.S., Natali, P.G., Ferrone, S., 1983. Cancer research, 43, 8, 35863590 .

Giacomini, P., Beretta, A., Nicotra, M., R., Ciccarelli, G., Martayan, A., Cerboni, C., et al., 1997. Tissue Antigens, 50, 555-566.

Giacomini. P., Giorda. E., Pera. C., Ferrara. G.B., 2001. Lancet Oncol, 2:658-658.

Konopsky V. N., Alieva E. V., 2007. Anal. Chem., 79 (12), 4729-4735.

Mahfoud, O.K., Rakovich, T.Y., Prina-Mello, A., Movia, D., Alvesc, F., and Volkovab, Y., 2014. RSC Adv, 4, 3422-3442.

Marques, R.C., Viswanathan, S., Nouws, H.P., Delerue-Matos, C., González-García M.B., 2014. Talanta, 129, 594-599.

Michelotti, F., Schmieder, S., Anopchenko, A., Munzert, P., Sinibaldi, A., Chandrawati, R., Rana, S., Sonntag, F., Occhicone, A., Napione, L., Stevens, M. M., Maillart, E., Hibti, F.-E., Frydman, C., Danz, N., 2016. Proc. of SPIE, 9750, 97501D-1-10.

Morales, A. J., Swairjo, M. A., and Schimmel, P., 1999. EMBO J., 18, 3475-3483.

Mucelli, S.P., Zamuner, M.,Tormen, M.,Stanta, G.,Ugo, P., 2008. Biosens.Bioelectron. 23,19001903.

Negro, A., Brar, B., Gu, Y., Peterson, K., Vale, W., and Lee, K.F., 2006. Proc. Natl. Acad. Sci. U. S. A., 103(43), 15889-15893. 
Nilvebrant, J., Åstrand, M., Georgieva-Kotseva, M., Björnmalm, M., Löfblom, J., Hober, S., 2014. PLOS ONE, 9, e103094.

O’Shannessy, D. J., Winzor, D. J., 1996. Anal. Biochem., 236, 275-283.

Ozato, K., Mayer, N.M., Sachs, D.H., 1982. Transplantation, 34(3), 113-120.

Patris, S., De Pauw, D., Vandeput, M., Huet, J., VanAntwerpen,P., Muyldermans, S.,Kauffmann, ,J.M, 2014. Talanta 130, 164-170.

Rizzo R., Danz N., Michelotti F., Maillart E., Anopchenko A., Wächter C., 2014. Opt. Express, 22 19 20223-214

Shi,Y., Huang,W., Tan,Y., Jin,X., Dua,R., Penuel,E., Mukherjee,A., Sperinde,J., Pannu, H., Chenna,A., DeFazio-Eli,L., Pidaparthi,S., Badal,Y., Wallweber,G., Chen, L., Williams,S., Tahir,H., Larson,J., Goodman,L., Whitcomb,J., Petropoulos,C., Winslow,J.,2009. DiagnMol. Pathol., 18, 11-21.

Shinn, M., Robertson, W.M., 2005. Sens. Act. B, 105, 2, 360-364.

Sinibaldi, A.; Rizzo, R.; Figliozzi, G.; Descrovi, E.; Danz, N.; Munzert, P.; Anopchenko, A.;and Michelotti, F., 2013. Opt. Express, 21, 20 23331-23344.

Sinibaldi, A.; Fieramosca, A.; Rizzo, R.; Anopchenko, A.; Danz, N.; Munzert, P.; Magistris, C.; Barolo, C.; Michelotti, F., 2014. Opt. Lett., 39, 10, 2947-2950.

Sinibaldi, A., Danz, N., Anopchenko, A., Munzert, P., Schmieder, S., Chandrawati, R., Rizzo, R., Rana, S., Sonntag, F., Occhicone, A., Napione, L., De Panfilis, S., Stevens, M.M., and Michelotti, F., 2015a. J. Lightw. Technol., 33, 16, 3385-3393.

Sinibaldi A., Anopchenko A., Rizzo R., Danz N., Munzert P., Rivolo P., Frascella F., Ricciardi S., Michelotti F., 2015b. Anal. Bioanal. Chem., 407, 3965-3974.

Toma K., Descrovi E., Toma M., Ballarini M., Mandracci P., Giorgis F., Mateescu A., Jonas U., Knoll W., Dostalek J., 2013. Biosens.Bioelectron. 43 108-114.

Yeh, P., Yariv, A., Hon, C-S., 1977. J. Opt. Soc. Am., 67, 4, 423-438.

Wang, Y., Brunsen, A., Jonas, U., Dostalek, J., and Knoll, W., 2009. Anal.Chem., 81, 9625-9632.

Zhang, Y., González, R.M., Zangar., R.C., 2011. BMCCancer, 11:69, 10.1186/1471-2407-11-69. 